\title{
The Stress State of a Finite Elastic Cylinder under Its Proper Weight
}

\author{
Filipchuk Anastasiia, Protserov Yuriy, Vaysfeld Natalya* \\ Odesa Mechnikov University, str. Dvoryanskaya, 2, 65082, Odesa, Ukraine. \\ * Corresponding author. Email: vaysfeld@onu.edu.ua \\ Manuscript submitted January 10, 2018; accepted October 12, 2018. \\ doi: 10.17706/ijapm.2019.9.1.65-71
}

\begin{abstract}
The stress state of a finite circular elastic cylinder under its own proper weight is evaluated in this paper. The edges of the cylinder are fixed. The circular surface is free from stress. To solve this problem, the finite Fourier's integral transformations were applied to the equilibrium equations and subjected boundary conditions. The stated problem was reduced to a one-dimensional vector boundary problem at the transformations' domain with regard to the unknown displacement's transformations. The apparatus of matrix differential calculations is used and the explicit solution of the vector boundary problem is constructed. These obtained formulas for the displacements have an unknown function which was found by solving the corresponding singular integral equation. The numerical results indicating the dependence of the cylinder's stress state on its geometrical parameters and proper weight were derived.
\end{abstract}

Key words: Finite cylinder, integral transformations, proper weight, singular integral equation.

\section{Introduction}

The problem of equilibrium of an elastic cylinder of finite length is one of the oldest in elasticity. A wide review of the papers and the ideas dedicated to this topic are given in paper [1]. The construction of general solutions in an axially symmetric case was made in work [2]. Steel oscillations of an elastic cylinder under an axisymmetric load applied on its upper edges are investigated in paper [3]. The work [4] presents the results of solving exact three-dimensional equations of the theory of elasticity with a longitudinal impact on the edges of the circle cylinder. Comparing the exact theoretical solutions with the calculations based on the elementary theory of the rods and its concluded that it is possible to reduce the problem to the one-dimensional. The comparison was made for the asymptotic solutions obtained in the work [5]. Numerical methods of solving problems of deformation of finite cylinders and rods are developed in paper [6], [7]. In the review [1] authors also stated that some problems of estimating a cylinder's stress state have yet to be solved due to their complexity. One such problem is proposed in this paper.

In this article we consider the 3-D problem for a finite-length isotropic elastic cylinder subjected to its own proper weight. Such a solution is important for the distribution of stress in cylinders exposed to such load in many areas of engineering and construction.

The novelty of the proposed approach is in the application of integral transformations directly to the equilibrium equations. It allows the construction of a one-dimensional boundary value problem in the transformations domain. This problem is solved exactly with the apparatus of matrix differential calculations. First, this approach was proposed and applied at [8], [9]. Its use reduces the problem to a 
singular integral equation which is solved with the help of the orthogonal polynomials method.

\section{The Statement of the Problem}

The elastic circular cylinder $0 \leq r \leq a,-\pi \leq \varphi \leq \pi,-l \leq z \leq l ; r, \varphi, z-$ is the cylindrical coordinate system), fixed at the edges $z= \pm l$ is considered. The lateral surface of the cylinder is free from stress.

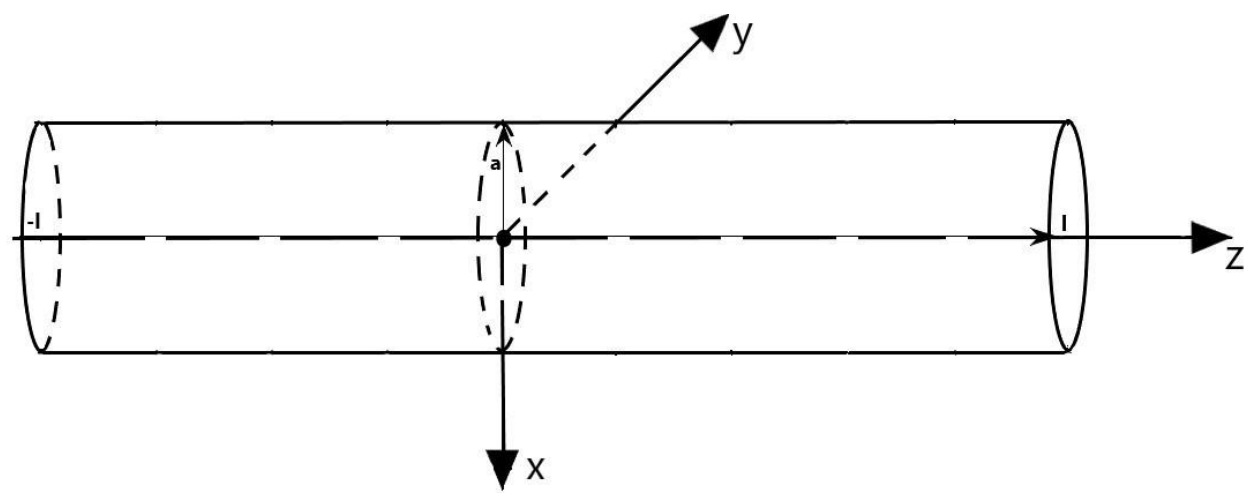

Fig. 1. The geometry of the problem.

The cylinder is deformed by its own weight. The cylinder's displacements should satisfy the equilibrium equations:

$$
\left\{\begin{array}{c}
U_{r}-\frac{1}{r^{2}} U_{r}-\frac{2}{r^{2}} \frac{\partial U_{\varphi}}{\partial \varphi}+\frac{2}{x-1} \frac{\partial \theta}{\partial r}=-\frac{\gamma}{G} \cos \varphi \\
U_{\varphi}-\frac{1}{r^{2}} U_{\varphi}-\frac{2}{r^{2}} \frac{\partial U_{r}}{\partial \varphi}+\frac{2}{x-1} \frac{1}{r} \frac{\partial \theta}{\partial r}=\frac{\gamma}{G} \sin \varphi \\
U_{z}+\frac{2}{x-1} \frac{\partial \theta}{\partial z}=0,
\end{array}\right.
$$

where $G$ is a shift module, $\gamma-$ a specific weight, $f=\frac{1}{r} \frac{\partial}{\partial r}\left(\mathrm{r} \frac{\partial \varphi}{\partial r}\right)+\frac{1}{r^{2}} \frac{\partial^{2} f}{\partial \varphi^{2}}+\frac{\partial^{2} \varphi}{\partial z^{2}}-$ is Laplace operator in the cylindrical coordinate system, $\theta=\frac{1}{r} \frac{\partial}{\partial r}\left(r U_{r}\right)+\frac{1}{r} \frac{\partial U_{\varphi}}{\partial \varphi}+\frac{\partial U_{z}}{\partial z}$ is a volume expansion, $x=3-4 \mu, \mu-$ Poisson's coefficient. The problem is symmetrical with regard to variable $z$, so it is sufficient to consider the area $0 \leq r \leq a,-\pi \leq \varphi \leq \pi, 0 \leq z \leq l$. Bearing that in mind, the boundary conditions will be written as

$$
\begin{gathered}
\left.\frac{\partial U_{r}}{\partial z}\right|_{z=0}=0,\left.\frac{\partial U_{\varphi}}{\partial z}\right|_{z=0}=0,\left.U_{z}\right|_{z=0}=0 ;\left.U_{r}\right|_{z=l}=0,\left.U_{\varphi}\right|_{z=l}=0,\left.U_{z}\right|_{z=l}=0 \\
\left.\sigma_{r}\right|_{r=a}=\left.2 G\left(\frac{\partial U_{r}}{\partial r}+\frac{3-x}{2(x-1)} \theta\right)\right|_{r=a}=0,\left.\tau_{r \varphi}\right|_{r=a}=\left.G\left(r \frac{\partial}{\partial r}\left(\frac{1}{r} U_{\varphi}\right)+\frac{1}{r} \frac{\partial U_{r}}{\partial \varphi}\right)\right|_{r=a}=0 \\
\left.\tau_{z \varphi}\right|_{r=a}=\left.G\left(\frac{\partial U_{z}}{\partial r}+\frac{\partial U_{r}}{\partial z}\right)\right|_{r=a}=0 .
\end{gathered}
$$

It is needed to derive the displacements and stresses satisfying the equations (1) subjected to the boundary conditions (2). 


\section{Reducing the Problem to a One Dimensional Boundary Value Problem and Its Solving}

The dimensionless variables were input with the formulas $\rho=\frac{r}{a}$ and $\xi=\frac{z}{l}$. The unknown displacements were searched with the form

$$
\begin{gathered}
U(r, \varphi, z)=U(\rho, \varphi, \xi)=U(\rho, \xi) \cos \varphi \\
V(r, \varphi, z)=V(\rho, \varphi, \xi)=V(\rho, \xi) \sin \varphi \\
W(r, \varphi, z)=W(\rho, \varphi, \xi)=W(\rho, \xi) \cos \varphi
\end{gathered}
$$

The finite integral Fourier's transformation with regard to variable $\xi$ reduce the stated problem to a one-dimensional boundary value problem in the transformations' domain. This problem is reformulated as a vector boundary problem:

$$
\left\{\begin{array}{c}
\overrightarrow{L y}(\rho)=\vec{f}(\rho) \\
V_{i}[\rho]=\gamma_{i}, \gamma=0, i
\end{array}\right.
$$

here $L$ is the differential matrix-operator of $3 \times 3$ order:

$$
L=\left(\begin{array}{ccc}
x\left(\begin{array}{ccc}
\left.D_{\rho}-\frac{4}{\rho^{2}}\right)-(x-1) b_{k}^{2} & D_{\rho}-\frac{2}{\rho} \frac{d}{d \rho} & 2 b_{k}\left(\frac{d}{d \rho}+\frac{1}{\rho}\right) \\
D_{\rho}+\frac{2}{\rho} \frac{d}{d \rho} & x D_{\rho}-(x-1) b_{k}^{2} & 2 b_{k}\left(\frac{d}{d \rho}-\frac{1}{\rho}\right) \\
-b_{k}\left(\frac{d}{d \rho}+\frac{2}{\rho}\right) & -b_{k} \frac{d}{d \rho} & (x-1)\left(D_{\rho}-\frac{1}{\rho^{2}}\right)-(x+1) b_{k}^{2}
\end{array}\right) \\
\vec{y}(\rho)=\left(\begin{array}{c}
W_{k}^{(1)}(\rho) \\
W_{k}^{(2)}(\rho) \\
W_{k}^{(3)}(\rho)
\end{array}\right), \vec{f}(\rho)=\left(\begin{array}{c}
(-1)^{k} \frac{2 \gamma a^{2}}{G v_{k}} \\
(-1)^{k} a^{2}(x+1) \chi(\rho)
\end{array}\right)
\end{array}\right.
$$

functions $W_{k}^{(i)}(\rho)$ are the transformations of the new unknown functions connected with the initial displacements by the correspondences

$$
\begin{gathered}
W_{k}^{(1)}(\rho)=U_{k}(\rho)+V_{k}(\rho) \\
W_{k}^{(2)}(\rho)=U_{k}(\rho)-V_{k}(\rho) \\
W_{k}^{(3)}(\rho)=W_{k}(\rho)
\end{gathered}
$$

$k$ - is the Fourier's parameter. Function $\chi(\rho)$ is the unknown function $\chi(\rho)=\left.\frac{\partial W}{\partial \xi}\right|_{\xi=1}$, 
$D_{\rho}(f)=\frac{1}{\rho}\left(\rho f^{\prime}\right)^{\prime}$.

The vector boundary problem (3) is solved with matrix differential calculations apparatus.

Before constructing the regular solution at $\rho=0$ of the homogeneous vector equation (3), one needs to construct the matrix solution of the corresponding equation

$$
L Y(\rho)=0,0<\rho<1
$$

where $Y(\rho)$ is a matrix $3 \times 3$ order. It can be checked that for a matrix

$$
H(\rho, s)=\left(\begin{array}{ccc}
I_{2}(\rho, s) & 0 & 0 \\
0 & I_{0}(\rho, s) & 0 \\
0 & 0 & I_{1}(\rho, s)
\end{array}\right)
$$

the equality $L H(\rho, s)=-H(\rho, s) M(s)$ is true. Here matrix $M(s)$ has the form:

$$
M(s)=\left(\begin{array}{ccc}
x s^{2}+(x-1) b_{k}^{2} & -s^{2} & 2 b_{k} s \\
-s^{2} & x s^{2}+(x-1) b_{k}^{2} & -2 b_{k} s \\
b_{k} s & -b_{k} s & (x-1) s^{2}+(x+1) b_{k}^{2}
\end{array}\right) \text {, }
$$

$b_{k}=a \vartheta_{k}, \quad \vartheta_{k}=\frac{\pi}{2}(2 k-1), \quad k=1,2, \ldots$. This matrix can be inversed:

$$
M^{-1}(s)=\frac{1}{\left(x^{2}-1\right)\left(s^{2}+b_{k}^{2}\right)^{2}}\left(\begin{array}{ccc}
x s^{2}+(x+1) b_{k}^{2} & s^{2} & -2 b_{k} s \\
s^{2} & x s^{2}+(x+1) b_{k}^{2} & 2 b_{k} s \\
-b_{k} s & b_{k} s & (x+1) s^{2}+(x-1) b_{k}^{2}
\end{array}\right) \text {. }
$$

The solutions of the matrix equation (4) are written as

$$
Y^{ \pm}(\rho)=\frac{1}{2 \pi} \prod_{c+} H(\rho, s) M^{-1}(s) d s
$$

Here $c_{+}$are the contours that cover all poles of the second order $s= \pm i b_{k}$. With the help of the contour integration we will find the exact form of the matrices (5). The derived solutions are complex-conjugated ones, so the solution of matrix equation (4) will be

$$
Y(\rho)=\operatorname{Re} Y^{ \pm}(\rho) \pm \operatorname{Im} Y^{ \pm}(\rho)
$$

Hence, the general solution of vector homogeneous equation (3) will be the vector:

$$
\bar{y}(\rho)=Y(\rho)\left(\begin{array}{l}
c_{1} \\
c_{2} \\
c_{3}
\end{array}\right),
$$

where $c_{i}, i=\overline{1,3}$ are the constants. To find the particular solutions of the equation (3) the fundamental 
matrix $\Phi(\rho, t)$ was constructed. Finally, the solution of the vector problem in the transformations domain has the form

$$
\bar{y}(\rho)=Y(\rho)\left(\begin{array}{l}
c_{1} \\
c_{2} \\
c_{3}
\end{array}\right)-\frac{1}{x^{2}-1} \int_{0}^{1} \Phi(\rho, t) \bar{f}(t) t d t
$$

The components of vector $\bar{y}(\rho)$ are constructed in explicit form.

$$
\begin{aligned}
& W_{k}^{(1)}(\rho)=\frac{1}{4 b_{k}\left(x^{2}-1\right)}\left\{\left[(2 x+3) I_{2}\left(b_{k} \rho\right)-b_{k} \rho I_{1}\left(b_{k} \rho\right)\right] B_{1}\left(b_{k}\right)+\left[-I_{2}\left(b_{k} \rho\right)+b_{k} \rho I_{1}\left(b_{k} \rho\right)\right] B_{2}\left(b_{k}\right)+\right. \\
& \left.+\left[4 I_{2}\left(b_{k} \rho\right)-2 b_{k} \rho I_{1}\left(b_{k} \rho\right)\right] B_{3}\left(b_{k}\right)\right\}+\frac{4 b_{k}(x+1) \alpha^{2}(-1)^{k}}{4 b_{k}\left(x^{2}-1\right)} \int_{0}^{1}\left\{\left[(2 x+3) I_{2}\left(b_{k} \rho\right)-b_{k} \rho I_{1}\left(b_{k} \rho\right)\right] D_{1 k}(t)+\right. \\
& \left.+\left[-I_{2}\left(b_{k} \rho\right)+b_{k} \rho I_{1}\left(b_{k} \rho\right)\right] D_{2 k}(t)+\left[4 I_{2}\left(b_{k} \rho\right)-2 b_{k} \rho I_{1}\left(b_{k} \rho\right)\right] B_{3 k}(t)\right\} \chi(t) t d t-\frac{2 \gamma a^{2} \alpha(-1)^{k}}{G b_{k}\left(x^{2}-1\right)} \times \\
& \times \int_{0}^{1} \Phi_{12}(\rho, t) d t-\frac{(x+1) \alpha^{2}(-1)^{k}}{\left(x^{2}-1\right)} \int_{0}^{1} \Phi_{13}(\rho, t) \chi(t) t d t . \\
& \quad W_{k}^{(2)}(\rho)=\frac{1}{4 b_{k}\left(x^{2}-1\right)}\left\{\left[-I_{0}\left(b_{k} \rho\right)-b_{k} \rho I_{1}\left(b_{k} \rho\right)\right] B_{1}\left(b_{k}\right)+\left[-(2 x+1) I_{0}\left(b_{k} \rho\right)+b_{k} \rho I_{1}\left(b_{k} \rho\right)\right] B_{2}\left(b_{k}\right)-\right. \\
& \left.\quad-2 b_{k} \rho I_{1}\left(b_{k} \rho\right) B_{3}\left(b_{k}\right)\right\}+\frac{(x+1) \alpha^{2}(-1)^{k}}{\left(x^{2}-1\right)} \int_{0}^{1}\left\{\left[-I_{0}\left(b_{k} \rho\right)-b_{k} \rho I_{1}\left(b_{k} \rho\right)\right] D_{1 k}(t)+\right. \\
& \left.+\left[-(2 x+1) I_{0}\left(b_{k} \rho\right)+b_{k} \rho I_{1}\left(b_{k} \rho\right)\right] D_{2 k}(t)-2 b_{k} \rho I_{1}\left(b_{k} \rho\right) D_{3 k}(t)\right\} \chi(t) t d t-\frac{2 \gamma a^{2} \alpha(-1)^{k}}{G b_{k}\left(x^{2}-1\right)} \times \\
& \quad \times \int_{0}^{1} \Phi_{22}(\rho, t) d t-\frac{(x+1) \alpha^{2}(-1)^{k}}{\left(x^{2}-1\right)} \int_{0}^{1} \Phi_{23}(\rho, t) \chi(t) t d t .
\end{aligned}
$$

Then $U_{k}(\rho)=\frac{1}{2}\left[W_{k}^{(1)}(\rho)+W_{k}^{(2)}(\rho)\right]$ and $V_{k}(\rho)=\frac{1}{2}\left[W_{k}^{(1)}(\rho)-W_{k}^{(2)}(\rho)\right]$.

$$
\begin{aligned}
& W_{k}^{(3)}(\rho)=W_{k}(\rho)=\frac{1}{4 b_{k}\left(x^{2}-1\right)}\left\{\left[-I_{1}\left(b_{k} \rho\right)+b_{k} \rho I_{0}\left(b_{k} \rho\right)\right] B_{1}\left(b_{k}\right)+\left[I_{1}\left(b_{k} \rho\right)-b_{k} \rho I_{0}\left(b_{k} \rho\right)\right] B_{2}\left(b_{k}\right)+\right. \\
& \left.+\left[2(x-1) I_{1}\left(b_{k} \rho\right)+2 b_{k} \rho I_{0}\left(b_{k} \rho\right)\right] B_{3}\left(b_{k}\right)\right\}+\frac{(x+1) \alpha^{2}(-1)^{k}}{\left(x^{2}-1\right)} \int_{0}^{1}\left\{\left[I_{1}\left(b_{k} \rho\right)-b_{k} \rho I_{0}\left(b_{k} \rho\right)\right]\left[D_{1 k}(t)+D_{2 k}(t)\right]\right\} d t .
\end{aligned}
$$

The application of inverse Fourier's transformations to these components leads to the displacement's formulas.

$$
\begin{aligned}
& U(\rho, \xi)=2 \sum_{k=1}^{\infty} U_{k}(\rho) \cos \left(\vartheta_{k} \xi\right) \\
& V(\rho, \xi)=2 \sum_{k=1}^{\infty} V_{k}(\rho) \cos \left(\vartheta_{k} \xi\right)
\end{aligned}
$$




$$
W(\rho, \xi)=2 \sum_{k=1}^{\infty} W_{k}(\rho) \sin \left(\vartheta_{k} \xi\right)
$$

The problem will be finally solved if the unknown function $\chi(\rho)$ can be found. All boundary conditions when $\xi=0$ are executed:

$$
\frac{\partial U}{\partial \xi}=0, \frac{\partial V}{\partial \xi}=0, \quad W=0 .
$$

From the boundary conditions when $\xi=1$ only two are satisfied:

$$
U=0, \quad V=0 .
$$

So, we should demand the third condition, satisfying:

$$
\left.W\right|_{\xi=1}=0
$$

After some transformations, a singular integral equation is obtained with regard to the unknown function $\chi(\rho)$. The solution of the equation is searched in the form

$$
\chi(\rho)=\left(1-\rho^{2}\right)^{\beta} \sum_{n=0}^{\infty} \mathrm{X}_{n} P_{n}^{0, \beta}\left(1-2 \rho^{2}\right)
$$

The solution is reduced to a linear algebraic system equation. The numerical analysis was done. The dependences of the cylinder's stress state on its proper weight, length and radius were investigated.

\section{Conclusion}

The problem for the finite circular cylinder under its proper weight is solved. The important dependences of the cylinder's stresses on its geometrical parameters were stated.

The approach proposed in this paper enables the application of this method to solve the dynamic problem for a cylinder under a steady-state loading.

\section{References}

[1] Meleshko, V., Tokovyy, Y., \& Barber, I. (2011). Axially symmetric temperature stresses in an elastic isotropic cylinder of finite length. Journal of Mathematical Science, 176(5), 646-669.

[2] Grinchenko, V., \& Meleshko, V. (1978). Axisymmetric vibrations of an elastic cylinder of finite length. Acoustic Journal, 24(6), 12-17.

[3] Makaryan, V., \& Simonyan, V. (1998). About a dynamic problem for a circular elastic cylinder of finite length. Mechanics. Proceedings of National Academy of Sciences of Armenia, 51(1), 26-31.

[4] Vales, F., Moravka, S., Brepta, R., \& Cerv, J. (1996). Wave propagation in a thick cylindrical bar due to longitudinal impact. JSME Int. J. A., 39(1), 60-70.

[5] Skalak, K. (1957). Longitudinal impact of a semiinfinite circular elastic bar. Trans. ASME. J. Appl. Mech., 24(1), 59-64.

[6] Bagenov, V., \& Chekmarev, D. (2001). Numerical methods for solving problems of the nonstationary dynamics of thin-walled structures. Mechanics of Solids, 5, 156-167. 
[7] Abramyan, B. (1954). To the problem of axisymmetric deformation of a circular cylinder. Reports of the Academy of Sciences of the Armenian SSR, 19(1), 23-28.

[8] Menshykov, O., Menshykova, M., \& Vaysfeld, N. (2017). Exact analytical solution for a pie-shaped wedge thick plate under oscillating load. Acta Mechanica, 228(12), 4435-4450.

[9] Popov, G., Protserov, Y., \& Gonchar, I. (2015). Exact solution of some axisymmetric Problems for Elastic Cylinders of Finite length taking into account Specific Weight. Journal of Intern Applied Mechanics, 51(4), 391-402.

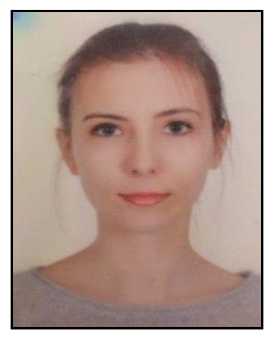

Anastasia Filipchuk is a third-year student of the Odessa National University of Applied Mathematics. She was born in Odessa on the sixteenth of January 1988. Her secondary education was received in the Odessa Mariinskaya Gymnasium in 2015. Her scientific interests are mathematical problems of elasticity.

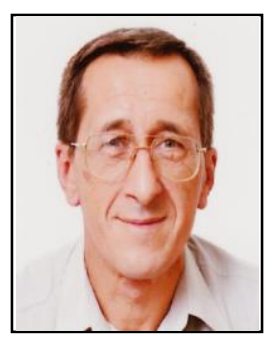

Yuriy Protserov is a senior lecturer of Mathematical Physics Methods Department at Odesa Mechnikov University, Ukraine. His scientific interests are focused on the mathematical problems of mechanics. He is author of more than 100 articles.

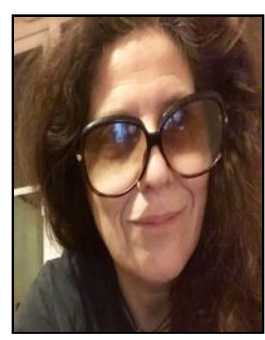

Natalya Vaysfeld is a head of Mathematical Physics Methods Department at Odesa Mechnikov University, Ukraine. Her scientific interests are focused on the mathematical problems of mathematical physics mixed problems for the areas with the discontinuities. She is author of more than 100 articles and 3 books. 